Document downloaded from:

http://hdl.handle.net/10251/44606

This paper must be cited as:

Hatami, S.; Ruiz García, R.; Andrés Romano, C. (2013). The distributed assembly permutation flowshop scheduling problem. International Journal of Production Research. 51(17):5292-5308. doi:10.1080/00207543.2013.807955.

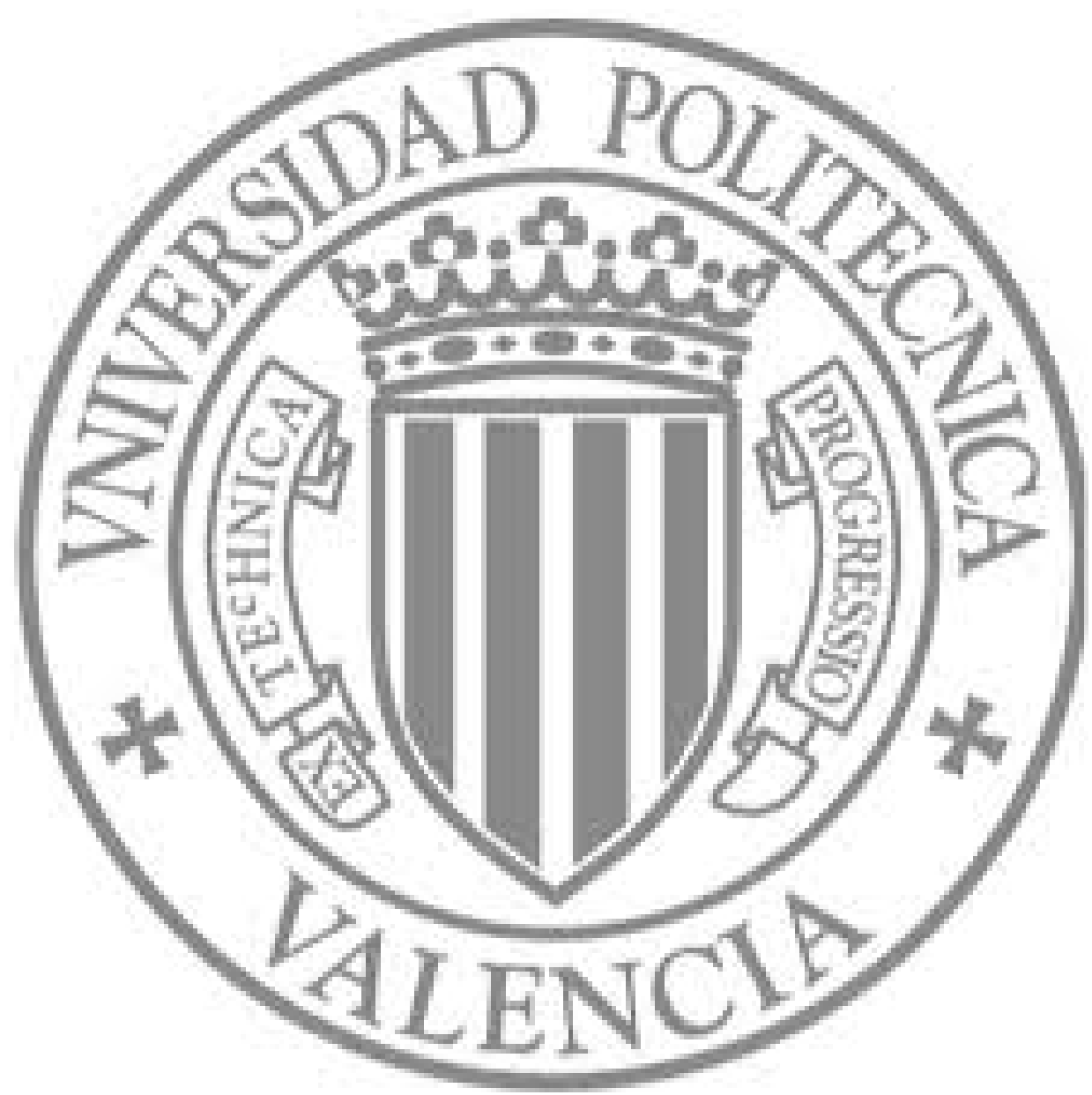

The final publication is available at

http://dx.doi.org/10.1080/00207543.2013.807955

Copyright Taylor \&amp;amp; Francis: STM, Behavioural Science and Public Health Titles 


\title{
The Distributed Assembly Permutation Flowshop Scheduling Problem
}

\author{
Sara Hatami ${ }^{\mathrm{a}}$, Rubén Ruiz ${ }^{\mathrm{b}, *}$, Carlos Andrés Romano ${ }^{\mathrm{c}}$ \\ ${ }^{a}$ Departamento de Estadística e Investigación Operativa, Facultad de Ciencias \\ Matemáticas, Universitat de València, Spain \\ ${ }^{b}$ Grupo de Sistemas de Optimización Aplicada, Instituto Tecnológico de Informática, \\ Ciudad Politécnica de la Innovación, Edifico 8G, Acc. B. Universitat Politècnica de \\ València, Camino de Vera s/n, 46021, València, Spain \\ ${ }^{c}$ Departamento de Organización de Empresas, Universidad Politècnica de \\ València,Camino de Vera s/n, 46021, València, Spain
}

\begin{abstract}
Nowadays, improving the management of complex supply chains is key to become competitive in the $21^{\text {st }}$ century global market. Supply chains are composed of multi-plant facilities that must be coordinated and synchronized to cut waste and lead times. This paper proposes a Distributed Assembly Permutation Flowshop Scheduling Problem (DAPFSP) with two stages to model and study complex supply chains. This problem is a generalization of the Distributed Permutation Flowshop Scheduling Problem (DPFSP) presented by Naderi and Ruiz (2010). The first stage of the DAPFSP is composed of $f$ identical production factories. Each one is a flowshop that produces jobs to be assembled into final products in a second assembly stage. The objective is to minimize the makespan. We present first a Mixed Integer Linear Programming model (MILP). Three constructive algorithms are proposed. Finally, a Variable Neighborhood Descent (VND) algorithm has been designed and tested by a comprehensive ANOVA statistical analysis. The results show that the VND algorithm offers good performance to solve this scheduling problem.
\end{abstract}

Keywords: Distributed assembly flowshop, Variable neighborhood descent

\footnotetext{
*Corresponding author

Email addresses: hatami@alumni.uv.es (Sara Hatami), rruiz@eio.upv.es (Rubén Ruiz), candres@omp.upv.es (Carlos Andrés Romano)
} 


\section{Introduction}

Assembly systems have been widely studied in the last decade given their practical interest and applications. An assembly flowshop is a hybrid production system where various production operations are independently and concurrently performed to make parts that are delivered to an assembly line (Koulamas and Kyparisis, 2001). In assembly systems, a wide variety of final products can be made from a given number of different assembled parts. Assembly programs represent relationships between the different parts which must be assembled from a set of suppliers.

Nowadays a single supplier or production factory is rare. As a matter of fact, production systems with more than one production center (named distributed manufacturing systems) are quite usual as they play an important role in practice (Moon et al., 2002). The benefits of distributed manufacturing systems include achieving higher product quality, lower production costs and fewer management risks (Wang, 1997; Kahn et al., 2004; Chan et al., 2005). From a manager's point of view, scheduling in distributed systems is more complicated than in single-factory scheduling problems. In singlefactory problems, the only objective is to find a job schedule for a set of machines, while an important additional decision in the distributed problem is allocating jobs to suitable factories. Therefore, two decisions have to be made; job allocation to factories and job scheduling at each factory. Different job allocations to different factories result in different production schedules, which consequently affects supply chain performance (Chan et al., 2005).

This paper contemplates flowshop scheduling as a production system for each factory or supplier in the distributed problem. The flowshop scheduling problem (FSP) is composed of a set of $M$ of $m$ machines where each job of a set $N$ of $n$ jobs must be processed in each machine. The number of operations per job is equal to the number of machines. The $i^{\text {th }}$ operation of each job is processed in machine $i$. Therefore, one job can start in machine $i$ only after it has been completed in machine $i-1$, and if machine $i$ is free. The processing times of each job in the machines are known in advance, non negative and deterministic. In FSPs, a number of assumptions are made (Baker, 1974): all jobs are available for processing at time 0; machines are continuously available (no breakdowns); each machine can process only one job at a time; each job can be processed in only one machine at a time; once the processing of a given job has started in a given machine, it cannot be interrupted and processing continues until completion (no preemption); setup 
times are sequence-independent and are either included in the processing times or ignored; infinite in-process storage is allowed.

In the FSP, there are $n$ ! possible job permutations for each machine. Therefore, the total number of solutions for a flowshop problem with $m$ machines is $(n !)^{m}$. To simplify the problem, it is assumed that all machines have the same job permutation. In other words, if one job is at the $j^{\text {th }}$ position on machine 1 , then this job has to be at the $j^{\text {th }}$ position on all other machines as well. With this simplifying assumption the FSP is referred to as Permutation Flowshop Scheduling Problem (PFSP) with $n$ ! possible solutions.

This paper studies the Distributed Assembly Flowshop Scheduling Problem (DAPFSP). It is a combination of the DPFSP and the Assembly Flowshop Scheduling Problem (AFSP), and consists of two stages: production and assembly. The first stage consists of a set $F$ of $f$ identical factories or production centers where a set $N$ of $n$ jobs have to be scheduled. All factories are capable of processing all jobs and each factory is a PFSP with a set $M$ of $m$ machines. Factories are assumed to be identical. Processing times are denoted by $p_{i j}, i \in M, j \in N$. The second stage is a single assembly factory with an assembly machine, $M_{A}$, which assembles jobs by using a defined assembly program to make a set $T$ of $t$ different final products. Each product has a defined assembly program; in other words, each product consists of some defined jobs. $N_{h}$ and $J_{j}$ are used, respectively, to represent product $h$ assembly program and the jobs that belong to the product $h$ assembly program, $N_{h}:\left\{J_{j}\right\}, j \in N_{h}$. Each product $h$ has $\left|N_{h}\right|$ jobs and job $j$ is needed for the assembly of one product. Therefore, $\sum_{h=1}^{t}\left|N_{h}\right|=n$. Product $h$ assembly can start only when all jobs that belong to $N_{h}$ have been completed in the factories. The considered objective is to minimize the makespan at the assembly factory.

The next section presents a short literature review. Section 3 provides a Mixed Integer Linear Programming (MILP) model to solve the considered problem. Section 4 introduces three constructive heuristics, while Section 5 presents an iterative method based on Variable Neighborhood Descent (VND) to improve results further. Section 6 describes a complete computational evaluation of the MILP model and proposed algorithms, where the performance of the proposed approaches is discussed in order to assess the influence of the number of jobs, machines, factories, products and some solver options on the results. Finally, Section 7 offers conclusions, remarks and venues for future research. 


\section{Literature review}

The DPFSP can be viewed as a generalized version of the PFSP. This problem is one of the most researched topics in the scheduling literature (Pinedo, 2012; Dong et al., 2009; Zobolas et al., 2009; Laha and Sarin, 2009; Vallada and Ruiz, 2010; Xu et al., 2011; Zhang and Li, 2011; Chen et al., 2012; Pan and Ruiz, 2012).

In the PFSP, more attention has been paid to makespan minimization. The practical implication is obvious: minimizing the makespan leads to the minimization of the total production run (Framinan et al., 2002). There are some proposed effective rules and algorithms for the PFSP (Johnson, 1954; Nawaz et al., 1983). A comprehensive review and evaluation has been made by Ruiz and Maroto (2005), Vallada et al. (2008) and Pan and Ruiz (2013).

Regarding the assembly scheduling problem, Lee et al. (1993) presented a three-machine assembly-type flowshop scheduling problem by considering makespan minimization as the objective function. In their considered model, each product is composed of two types of jobs, where type $a$ and $b$ are processed by machine $M_{a}$ and $M_{b}$, respectively, and machine $M_{2}$ assembles the two jobs into a product. These authors also present a branch-and-bound solution scheme and an approximate solution procedure. Later, Potts et al. (1995) extended the model of Lee et al. (1993) by considering $m$ parallel production machines instead of the first two production machines. They apply the compact vector summation technique to find approximated solutions with worse-case absolute performance guarantees. Hariri and Potts (1997) developed a branch-and-bound algorithm for the same model as Potts et al. (1995). Moreover, Tozkapan et al. (2003) considered a two-stage assembly scheduling problem by minimizing the total weighted flow time as an objective function. They developed a lower bound and a dominance criterion, and incorporated them into a branch-and-bound procedure. They also presented a heuristic procedure to find an initial upper bound. Al-Anzi and Allahverdi (2006) addressed the model presented by Tozkapan et al. (2003) and minimized the total completion time of all the jobs. They used metaheuristics to solve their model and proposed simulated annealing (SA), tabu search (TS), and hybrid tabu search heuristics for general cases.

Despite the innumerable literature related to PFSP and AFSP, there are few studies about the distributed problems. Jia et al. (2002) reported a webbased system to enable production scheduling (a job shop problem) for the distributed manufacturing environment and a Genetic Algorithm (GA) was 
adopted to solve the problem. Jia et al. (2003) presented a modified GA to deal with distributed job shop scheduling problems. Later, Jia et al. (2007) proposed a new approach to determine good combinations of factories to manufacture jobs. An adaptive GA for distributed scheduling problems was proposed by Chan et al. (2005). The same authors proposed a GA with dominant genes for solving distributed scheduling problems in an FMS environment in Chan et al. (2006a). Furthermore, Chan et al. (2006b) proposed a GA to deal with distributed flexible manufacturing system (FMS) subject to machine maintenance constraints. Naderi and Ruiz (2010) introduced the DPFSP for the first time. They developed six different MILPs for the considered problem and proposed two simple factory assignment rules and 14 heuristics based on dispatching rules, effective constructive heuristics and VND methods. Liu and Gao (2010) proposed an electromagnetism-like mechanism (EM) algorithm for the same problem. The same authors, in Gao and Chen (2011a) proposed a GA-based algorithm, denoted by GA-LS, Gao and Chen (2011b) a constructive heuristic algorithm enhanced with a dispatching rule, Gao et al. (2012b) a knowledge-based genetic algorithm and Gao et al. (2012a) a Variable Neighborhood Descent (VND) algorithm.

To the best of our knowledge, no further literature exists on DAPFSP, so this is the first effort that considers the assembly flowshop problem in a distributed manufacturing setting.

\section{Mixed Integer Linear Programming model}

A mathematical model is an abstract and good approach that uses mathematical language to describe in detail a problem. There are many papers related to the flowshop problem which use MILP modeling; for example, we can cite Stafford et al. (2005); Tseng and Stafford (2008); Ching-Jong and Li-Man (2008) and Naderi and Ruiz (2010), to name just a few.

We first define the model indexes, parameters and variables in Table 1 , and present the MILP afterwards. The proposed MILP model is inspired by the fifth mathematical model that is presented in Naderi and Ruiz (2010) for the DPFSP that was shown to outperform the other models tested in that paper.

The objective function of the model is to minimize a makespan: $\operatorname{Min} C_{\max }$ 


\begin{tabular}{ll}
\hline Index & Description \\
\hline$k, j$ & denotes jobs, $k, j=0,1, \ldots, n$, where 0 presents a dummy job \\
$i$ & denotes machines at each factory, $i=1, \ldots, m$ \\
$l, s$ & denotes products, $l, s=0,1, \ldots, t$, where 0 presents a dummy product \\
$M$ & A sufficiently large positive number \\
\hline Parameters & Description \\
\hline$n$ & number of jobs \\
$m$ & number of machines \\
$f$ & number of factories \\
$t$ & number of products \\
$p_{i j}$ & processing time of job $j$ on machine $i$ \\
$p p_{s}$ & processing time of product $s$ at the assembly stage \\
$G_{j s}$ & Binary parameter equal to 1 if job $j$ belongs to product $s$, and 0 otherwise \\
\hline Variable & Description \\
\hline$X_{k j}$ & binary variable equal to 1 if job $k$ is an immediate predecesor of job $j$ \\
$Y_{l s}$ & binary variable equal to 1 if product $l$ is an immediate predecesor of product $s$ \\
$C_{i j}$ & completion time of job $j$ on machine $i$ \\
$C A_{s}$ & completion time of product $s$ on assembly stage \\
$C_{\text {max }}$ & makespan \\
\hline
\end{tabular}

Table 1: indexes, parameters and variables used in MILP mathematical model.

$$
\begin{gathered}
\sum_{k=0, k \neq j}^{n} X_{k j}=1 \quad \forall j \\
\sum_{j=0, k \neq j}^{n} X_{k j} \leq 1 \quad \forall k \\
\sum_{j=1}^{n} X_{0 j}=f \\
\sum_{k j}+X_{j k} \leq 1 \quad X_{k 0}=f-1 \\
\forall j \in\{1, \ldots, n-1\}, j>k \\
C_{i j} \geq C_{i-1 j}+p_{i j} \quad \forall i, j \\
\sum_{l=0, l \neq s}^{t} Y_{l s}=1
\end{gathered}
$$




$$
\begin{array}{rr}
\sum_{s=1, l \neq s}^{t} Y_{l s} \leq 1 \quad & \forall l \\
Y_{l s}+Y_{s l} \leq 1 \quad \forall l \in\{1, \ldots, t-1\}, s>l \\
C A_{s} \geq\left(C_{m j} \cdot G_{j s}\right)+p p_{s} & \forall j, s \\
C A_{s} \geq C A_{l}+p p_{s}+\left(Y_{l s}-1\right) \cdot M & \forall l, s \\
C_{\max } \geq C A_{s} \quad \forall s \\
X_{k j} \in\{0,1\} \quad \forall k, j, k \neq j \\
Y_{l s} \in\{0,1\} \quad \forall l, s, l \neq s \\
C_{i j} \geq 0 \quad \forall i, j \\
C A_{s} \geq 0 \quad \forall s
\end{array}
$$

Note that $C_{0 j}=C A_{0}=0, \forall j$. Constraint set (1) controls and ensures that each job must have exactly one predecessor. Constraint set (2) indicates that each job has one succeeding job at the most. Constraint set (3) enforces that dummy job 0 has to have $f$ predecessor in the final sequence. Constraint set (4) also enforces that dummy job 0 must be a successor $f-1$ times (there is no dummy job at the end of the sequence). Constraint set (5) controls and ensures that a job cannot be both a predecessor and successor of another job at the same time. Constraint set (6) enforces the processing of job $j$ in machine $i$ when the processing at machine $i-1$ is completed. Constraint set (7) determines that if job $j$ is placed immediately after job $k$, its processing at machine $i$ cannot start before the processing of job $k$ in machine $i$ finishes. Constraints (8) and (9) force that each product should have one predecessor and at most one succeeding product in the assembly factory, respectively, constraint (10) controls that a product cannot be both a predecessor and a successor of another product at the same time. Constraint (11) implies that each product $h$ cannot begin its assembly before all the jobs in its assembly program are completed in the last machine $m$. Constraint set (12) determines that if product $s$ is placed immediately after product $l$, its processing on assembly machine cannot start before the processing of product $l$ in assembly machine finishes. Constraint (13) defines the makespan, while constraints (14)-(17) define the domain of the decision variables.

The significant point of this model is that there is no index for factories. Sequence-based variables are hence used with a set of $f$ dummy jobs. 
These dummy jobs divide all the jobs into subsequences and assign them to each factory (i.e., all jobs placed between the first dummy job and the second dummy job belong to the first factory, and so on). For example, if one of the possible solutions for a problem with $n=8$ and $f=3$ is $X_{0,2}=X_{2,3}=X_{3,5}=X_{5,0}=X_{0,6}=X_{6,1}=X_{1,4}=X_{4,0}=X_{0,7}=X_{7,8}=1$, then the sequence is $\{0,2,3,5,0,6,1,4,0,7,8\}$, where partial job sequences $\{2,3,5\},\{6,1,4\}$ and $\{7,8\}$ are assigned to factories 1,2 and 3 , respectively.

\section{Heuristic methods}

As mentioned in the paper of Naderi and Ruiz (2010), the DPFSP is an NP-Complete problem (if $n>f$ ); accordingly, the DAPFSP with an additional assembly stage as a further stage is certainly a NP-Complete problem. Therefore, it is necessary to develop a heuristic approach to solve large-sized problems. In order to solve instances of realistic size in this problem, three constructive simple heuristics are proposed.

For the assignment of jobs to factories, the two rules, of Naderi and Ruiz (2010) are used.

1. Assign job $j$ to the factory which has the lowest current $C_{\max },\left(\mathrm{NR}_{1}\right)$.

2. Assign job $j$ to the factory which has the lowest $C_{\max }$ after including job $j,\left(\mathrm{NR}_{2}\right)$.

Using these two factory allocation rules, three heuristics are presented to schedule jobs.

\subsection{Heuristic 1}

We first introduce some necessary notation. An example with $n=9$, $m=2, f=2$ and $t=3$, this is, 9 jobs, 2 factories with a flowshop of two machines each and three products to assemble, is employed to explain expressions and heuristics in detail. Table 2 shows the processing times of the jobs and assembly processing times of products. The products' assembly programs are: $N_{1}=\{3,4,6\}, N_{2}=\{1,2,8,9\}$ and $N_{3}=\{5,7\} . \pi$ represents a product sequence, e.g., $\pi:\{1,3,2\}$ is a possible product sequence for the given example. As mentioned before, each product $h$ is made up of $\left|N_{h}\right|$ jobs and $\pi_{h}$ is the partial job sequence of product $h$, e.g., $\pi_{1}:\{6,4,3\}$, $\pi_{2}:\{1,9,8,2\}$ and $\pi_{3}:\{7,5\}$. A complete job sequence, $\pi_{T}$, is constructed 
by putting together all partial job sequences, following the product sequence $\pi$, e.g., $\pi_{T}:\{6,4,3,7,5,1,9,8,2\}$.

The shortest processing time (SPT) is a well-known dispatching rule for the PFSP. In the SPT, the job with the shortest processing time is processed first. This rule tends to reduce the work-in-process inventory, the average throughput time, and average job lateness (Vollmann et al., 2005). Hence the SPT is used to determine the product sequence in the assembly machine.

Heuristic 1 begins by applying the SPT rule for the assembly operation times to obtain $\pi$. A heuristic which is based on Framinan and Leisten (2003) heuristic (FL) is applied on the jobs that belong to a given product, to obtain a good partial job sequence for each product. The heuristic evaluates the completion times of the jobs that belong to product $h$. Set $R_{h}$ is made by sorting jobs in ascending order of completion times. The first two jobs of $R_{h}$ are selected and inserted into $S_{h}$. When there are only two jobs in $S_{h}$, all pairwise exchanges are checked and $S_{h}$ is updated with the one that results in the best makespan. The next step is removing the third job in $R_{h}$ and inserting it in all possible positions of $S_{h}$. The sequence with the best makespan will be selected. All possible sequences by carrying out pairwise exchanges between jobs are evaluated again. The process continues until all jobs have been considered. $S_{h}$ is the partial job sequence for product $h,\left(\pi_{h}\right)$. $\pi_{T}$ is constructed by putting together all $\pi_{h}$ and jobs are assigned to factories from $\pi_{T}$ by using $\mathrm{NR}_{1}$ or $\mathrm{NR}_{2}$, which respectively result in the $\mathrm{H}_{11}$ or $\mathrm{H}_{12}$ heuristics.

Pseudocode 1 explains heuristics $\mathrm{H}_{11}$ and $\mathrm{H}_{12}$ in detail:

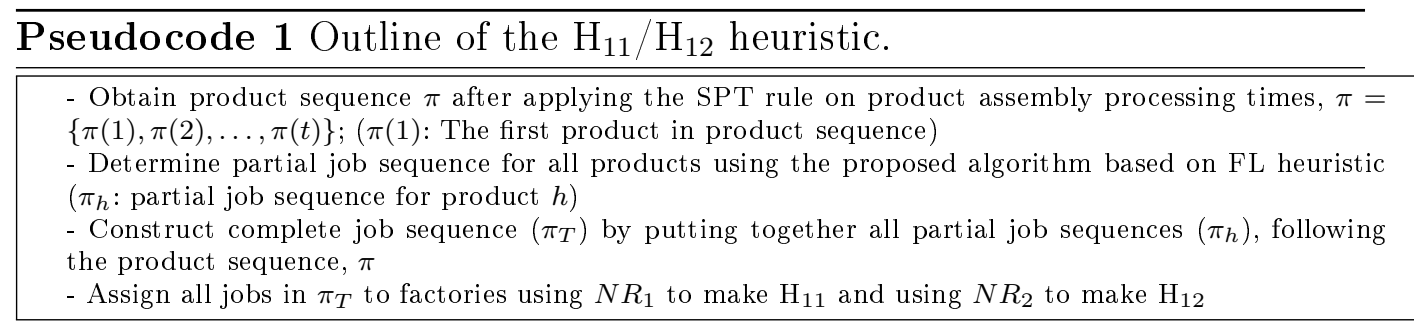

Let us now apply proposed heuristics to the example. $\pi:\{1,3,2\}$ is the product sequence obtained after applying the SPT rule to the assembly processing times of the products. The next step is to find a good partial job sequence for each product. As mentioned before, each product has a defined assembly program that includes a defined set of $\left|N_{h}\right|$ jobs. Completion time 


\begin{tabular}{cccccccccc}
\hline \multicolumn{10}{c}{ Jobs } \\
\hline Machines & 1 & 2 & 3 & 4 & 5 & 6 & 7 & 8 & 9 \\
\hline$M_{1}$ & 1 & 5 & 7 & 9 & 9 & 3 & 8 & 4 & 2 \\
\hline$M_{2}$ & 3 & 8 & 5 & 7 & 3 & 4 & 1 & 3 & 5 \\
\hline \multicolumn{1}{c}{ Product 1} & Product 2 & Product 3 \\
\hline$M_{A}$ & 6 & \multicolumn{1}{c}{19} & \multicolumn{1}{c}{12} \\
\hline
\end{tabular}

Table 2: Processing times of the jobs and assembly processing times of the products for the example.

for each job at the production stage is the summation of each job processing times on all machines, $\sum_{i=1}^{m} p_{i j}$. Therefore, completion times for set of jobs of the product $1, N_{1}=\{3,4,6\}$ are $C_{23}=12, C_{24}=16, C_{26}=7$. Set $R_{1}$ is obtained by arranging jobs in an increasing completion time order; $R_{1}=\{6,3,4\}$. The first two jobs of $R_{1}$ are selected and included into $S_{1}$. All possible sequences resulting from pairwise exchanges of the first two jobs in $S_{1}$ are calculated: $\{6,3\}$ and $\{3,6\}$ which result in makespans values of 15 and 16, respectively. The sequence with the minimum makespan is $S_{1}:\{6,3\}$. The third job in $R_{1},(4)$ is inserted into all possible positions of $S_{1}$. The obtained partial job sequences are: $\{4,6,3\},\{6,4,3\}$ and $\{6,3,4\}$ and their makespans in the production stage are: 25, 24, 26, respectively. As a result, the second is the best position for job 4 and $S_{1}$ is updated to $\{6,4,3\}$. In the next step, general pairwise exchanges are carried out on the updated $S_{1}$; hence, the partial job sequences are: $\{4,6,3\},\{6,3,4\}$ and $\{3,4,6\}$ and, subsequently, their makespans in the production stage are, 25, 26,27 , respectively. If a better makespan is obtained, then $S_{1}$ is updated. This process continues until all jobs have been inserted into $S_{1} \cdot \pi_{1}$ is the final updated $S_{1}$, which is equal to $\{6,4,3\}$. By following the same method, the partial job sequences for the other products are: $\pi_{2}=\{1,9,8,2\}$ and $\pi_{3}=\{5,7\}$ with partial makespans of 20 and 18, respectively. Hence $\pi_{T}$ is $\{6,4,3,5,7,1,9,8,2\}$. The final step is to assign jobs in $\pi_{T}$ to factories by using $\mathrm{NR}_{1} / \mathrm{NR}_{2}$ to obtain $\mathrm{H}_{11} / \mathrm{H}_{12} . C_{\max }$ of $\mathrm{H}_{11}$ and $\mathrm{H}_{12}$ are 55 and 53 , respectively. The Gantt chart of the considered example after applying $\mathrm{H}_{11}$ is shown in Figure 1.

\subsection{Heuristic 2}

The idea of the second heuristic is to give priority to products whose jobs are completed in the production stage sooner. This concept is noted as the 


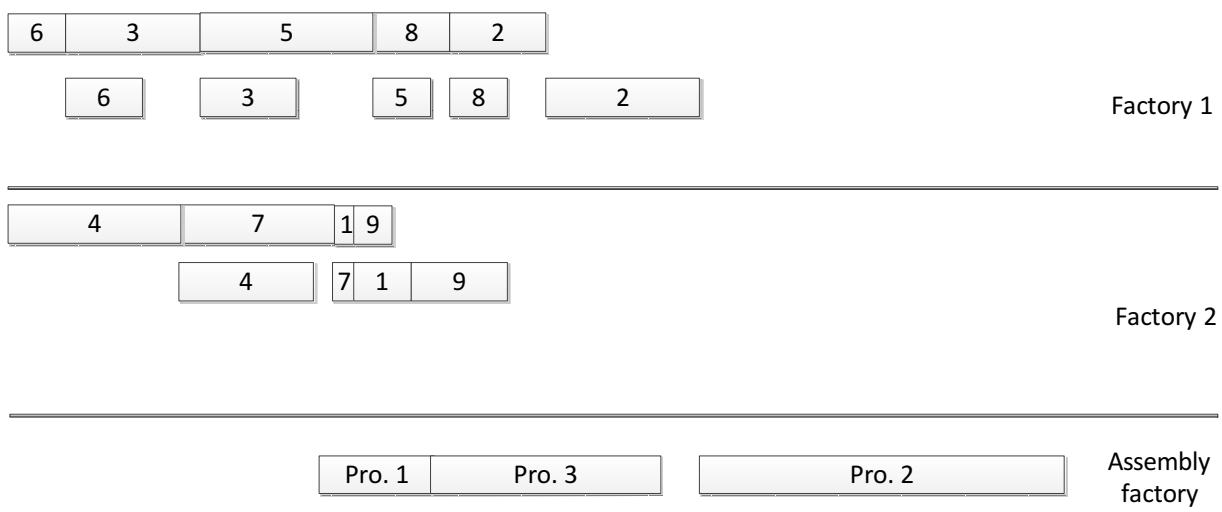

Figure 1: Gantt chart of $\mathrm{H}_{11}$ for the example.

earliest start time to assemble product $h, E_{h}$. The procedure that is used in $\mathrm{H}_{11}$ and $\mathrm{H}_{12}$ to find partial job sequences of products $\left(\pi_{h}\right)$ also is used in heuristic 2. $E_{h}$, is calculated by using $\mathrm{NR}_{1}$ or $\mathrm{NR}_{2}$ to assign jobs in each partial job sequence to factories. $\pi$ is built by sorting $E_{h}$ in ascending order. A detailed explanation is shown in Pseudocode 2.

\section{Pseudocode 2 Outline of the $\mathrm{H}_{21} / \mathrm{H}_{22}$ heuristic.}

- Determine partial job sequences of products using proposed algorithm based on FL heuristic $\left(\pi_{h}\right.$ : partial job sequence for product $h$ )

- Calculate the earliest start time to assemble each product $h, E_{h}$, using $\mathrm{NR}_{1}$ and $\mathrm{NR}_{2}$ to assign jobs of the partial job sequences, respectively for $\mathrm{H}_{21}$ and $\mathrm{H}_{22}$

- Sort $E_{h}$ in ascending order for all the products to obtain product sequence, $\pi:\{\pi(1), \pi(2), \ldots, \pi(t)\}$

- Construct complete job sequence $\left(\pi_{T}\right)$ by putting together all partial job sequences $\left(\pi_{h}\right)$, following the product sequence, $\pi$

- Assign all jobs of $\pi_{T}$ to factories using $N R_{1}$ to make $\mathrm{H}_{21}$ and using $N R_{2}$ to make $\mathrm{H}_{22}$

The last example data is also used to clarify the second proposed heuristic. $E_{h}$ is calculated by applying job assignment rules $\left(\mathrm{NR}_{1}\right.$ for the $\mathrm{H}_{21}$ and $\mathrm{NR}_{2}$ for the $\mathrm{H}_{22}$ ) for the partial job sequence of product $h$. Therefore, the earliest start times for assembling products by considering $\mathrm{NR}_{2}$ are $E_{1}=15$, $E_{2}=15$ and $E_{3}=12$. The product sequence $\pi$ is obtained by sorting $E_{h}$ in ascending order, $\pi:\{3,2,1\}$. As a result, the complete job sequence, $\pi_{T}$, will be: $\{5,7,1,9,8,2,6,4,3\}$. The final results of $C_{\max }$ for $\mathrm{H}_{21}$ and $\mathrm{H}_{22}$ are equal to 51 and 50 , respectively. 


\subsection{Heuristic 3}

The third proposed heuristic is similar to the second one. The difference is in the construction of the partial job sequences of each product $\left(\pi_{h}\right)$. While heuristic 2 uses a heuristic based on FL, heuristic 3 employs the more simple SPT rule. Our intention is to test if a simpler constructive heuristic gives similar results.

Table 3 shows the $C_{m j}$ of the jobs, the partial job sequence for each product, after applying the SPT rule and $E_{h}$ of product $h$ in the columns for the example.

\begin{tabular}{ccccc}
\hline Product $(h)$ & Job $(j)$ & $C_{2 j}$ & $\pi_{h}$ & $E_{h}$ \\
\hline \multirow{2}{*}{1} & 3 & 12 & & \\
& 4 & 16 & $6,3,4$ & 19 \\
& 6 & 7 & & \\
\hline \multirow{2}{*}{2} & 1 & 4 & & \\
& 2 & 13 & $1,9,8,2$ & 15 \\
& 8 & 7 & & \\
\hline \multirow{2}{*}{3} & 9 & 7 & & 12 \\
& 5 & 12 & 7,5 & \\
\hline
\end{tabular}

Table 3: Job completion times on the last machine of production stage, products partial job sequence and earliest start time for assembling each product for the example.

Product sequence $\pi$ is $\{3,2,1\}$ after sorting $E_{h}$ in ascending order. The complete sequence $\pi_{T}$ after putting together the partial jobs sequences of each product is: $\{7,5,1,9,8,2,6,3,4\}$. After applying $\mathrm{NR}_{1}$ to this sequence we obtain a $C_{\max }$ of 51 . The $C_{\max }$ for $\mathrm{NR}_{2}$ is 50 .

\section{Variable Neighborhood Descent (VND)}

We now present a Variable Neighborhood Descent (VND) method (Hansen and Mladenovic, 2001). VND is an enhanced local improvement strategy based on the systematic exploration of different neighborhood structures $N_{1}, \ldots, N_{q}$. A VND starts with the first structure $N_{1}$ by performing a local search until no further improvements are possible. From this local optimum, it continues the local search with neighborhood structure $\mathrm{N}_{2}$. If an improved solution is found with this structure, the VND goes back to $N_{1}$; otherwise, it continues with $N_{3}$, and so forth. If the last structure $N_{q}$ has been applied and no further improvements are possible, the solution represents a local optimum with respect to all neighborhood structures and the VND terminates. 


\subsection{Solution representation and VND initialization}

In order to represent a solution, a complete sequence of all jobs $\pi_{T}$ is considered, like in the PFSP. We limit the representation so that all jobs from a product are never separated. The jobs in the complete sequence are assigned to factories using $\mathrm{NR}_{1}$ or $\mathrm{NR}_{2}$. An example of a solution representation can be: $\{6,4,3,1,9,8,2,5,7\}$ which is a equal to product sequence of $\{1,2,3\}$ with respect to the last example.

The VND approach needs an initial solution. Although a random solution can be used as an initial solution, it is better to use heuristics (Naderi and Ruiz, 2010; Vallada and Ruiz, 2010; Ruiz and Stützle, 2007). Our approach uses the six proposed constructive heuristics to obtain the initial solution. Later we will test six VND versions, each one starting from the result of each heuristic.

\subsection{Neighborhoods and acceptance criterion}

Our proposed VND heuristic employs two neighborhood structures, and both are applied to the complete sequence $\pi_{T}$.

The first is referred to as $L S_{P}$ and is a product local search. It attempts to improve the objective function by examining different product sequences. $L S_{P}$ works as follows: 1) It provides a list of product sequences by removing a single product from $\pi$ and inserting it in all the possible $t-1$ positions of current $\pi ; 2$ ) It evaluates the list of obtained product sequences by converting them into $\pi_{T}$ and assigning the jobs of $\pi_{T}$ to factories via $\mathrm{NR}_{1}$ or $\mathrm{NR}_{2} ; 3$ ) If one of the obtained $\pi$ in the list has a better $C_{\max }$, then $\pi$ is updated to the better product sequence and all the products are reinserted again (a local search until a local optimum), otherwise the search continues with the next product.

The second neighborhood is $L S_{J}$, tries to find different partial job sequences for each product to improve the objective function. $L S_{J}$ works as follows: 1) $L S_{J}$ starts with the first product $h$, then the local search starts by removing the first job of $\pi_{h}$ and inserting it in all the possible $\left|N_{h}\right|-1$ positions of $\pi_{h}$;2) Evaluate $\pi_{T}$ with all the newly obtained partial job sequences for product $h ; 3$ ) If a better objective function is obtained, then $\pi_{h}$ is updated and all jobs in $\pi_{h}$ are reinserted again until a local optimum is found. Otherwise, the search continues with the next job in $\pi_{h}$;4) $L S_{J}$ will continue with the next product until all products have been considered.

Pseudocodes 3 and 4 show the product and the job local search, respectively. 

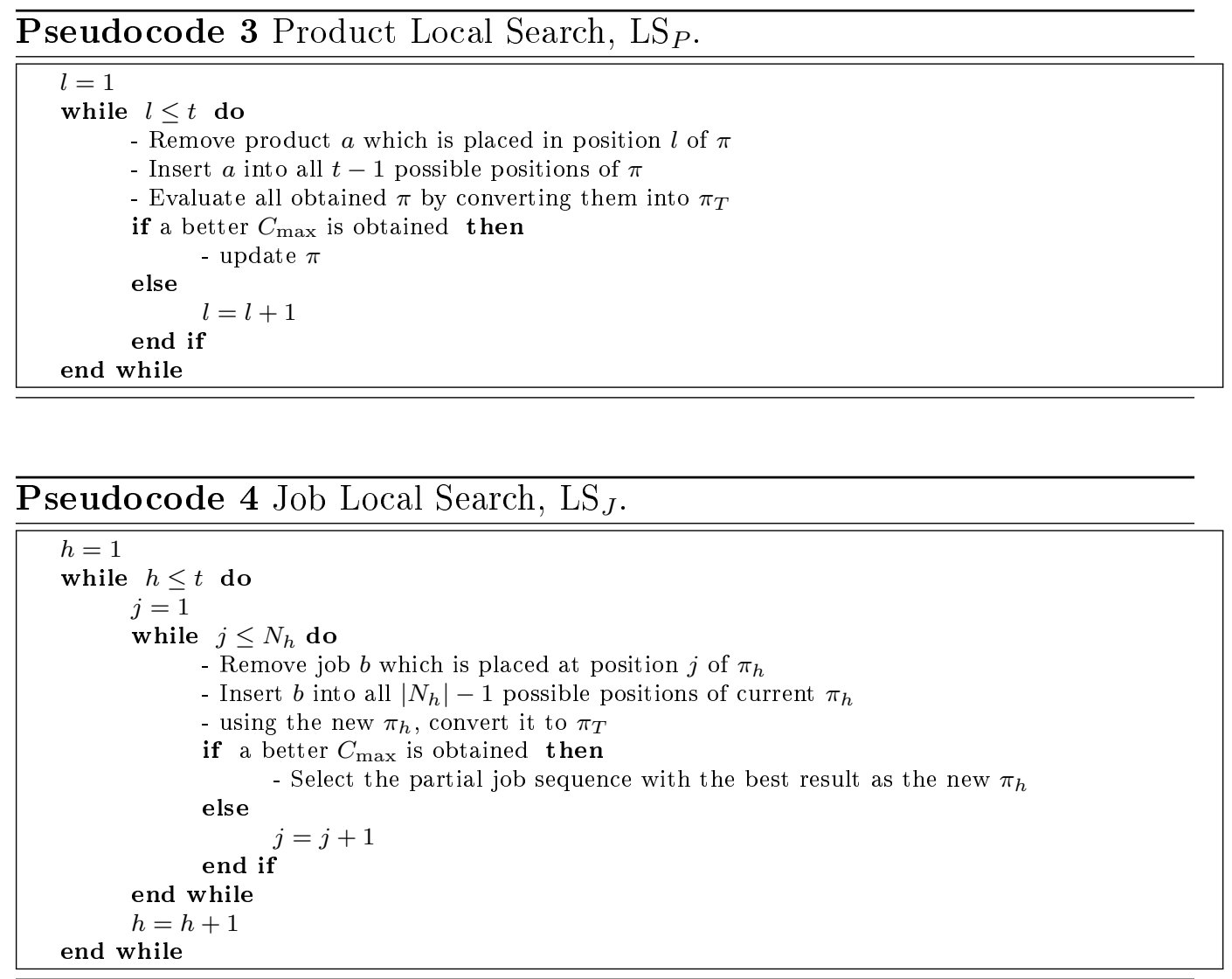

\section{Computational evaluation}

Two complete sets of instances have been generated to test the MILP model and the proposed heuristics. Due to the complexity of the problem, and given the number of different characteristics considered, four instance factors and three test factors are combined at the levels provided in Table 4 for small instances. The test factors are: two commercial solver packages (Solver) are used as solving tools, the number of CPU threads (Thread), where we have tested 1 thread (serial computing) and 2 threads (parallel computing) and a time limitation TimeLimit for the stopping criterion. The heuristics are also tested in a set of larger instances, which differ in the factors as listed in Table 5.

Processing times in the production stage are fixed to $U[1,99]$ as it is usual in the scheduling literature. The assembly processing times depend on the number of jobs assigned to each product $h$ as $U\left[1 \times\left|N_{h}\right|, 99 \times\left|N_{h}\right|\right]$. The total 


\begin{tabular}{llll}
\hline Instance factor & Symbol & Number of levels & Values \\
\hline Number of jobs & $n$ & 5 & $8,12,16,20,24$ \\
Number of machines & $m$ & 4 & $2,3,4,5$ \\
Number of factories & $f$ & 3 & $2,3,4$ \\
Number of products & $t$ & 3 & $2,3,4$ \\
\hline Test factor & Symbol & Number of levels & Values \\
\hline Solver & Solver & 2 & CPLEX 12.3, GUROBI 4.6.1 \\
Thread & Thread & 2 & Serial computing (1), Parallel computing (2) \\
Time limitation & TimeLimit & 2 & $900 s, 3600 s$ \\
\hline
\end{tabular}

Table 4: Instance and test factors for the small instances.

\begin{tabular}{llll}
\hline Instance factor & Symbol & Number of levels & Values \\
\hline Number of jobs & $n$ & 3 & $100,200,500$ \\
Number of machines & $m$ & 3 & $5,10,20$ \\
Number of factories & $f$ & 3 & $4,6,8$ \\
Number of products & $t$ & 3 & $30,40,50$ \\
\hline
\end{tabular}

Table 5: Instance factors for the large instances.

number of combinations in the small and large instances are $5 \times 4 \times 3^{2}=180$ and $3^{4}=81$, respectively. There are five replications per combination for small instances and ten replications for every large combination. Therefore, the total number of instances is 900 and 810, respectively. All the instances are available at http://soa.iti.es.

\subsection{MILP model evaluation}

A linear programming model has been constructed for each small instance. It is solved with all the combinations of the test factors, using CPLEX 12.3 and GUROBI 4.6.1 solvers, serial and parallel computing and two time limits (900s and 3600s). All the tests are carried out in a high performance computing cluster with 30 blades, each one containing 16 GBytes of RAM memory and two Intel XEON E5420 processors running at $2.5 \mathrm{GHz}$. Note that each processor has 4 physical computing cores ( 8 per blade). The 30 blade servers are used only to divide the workload and experimentations. Experiments are carried out in virtualized Windows XP machines, each with one virtualized processor with two cores and 2 GB of RAM memory.

A categorical variable named "response type" with two values, 0 and 1 , is reported. Value 0 means that an optimum solution is found in the given time with $C_{\max }$ value as a result, and 1 means that in $900 \mathrm{~s}$ or $3600 \mathrm{~s}$, a 
feasible integer solution is found and reported, but it has not been proven to be optimal. Moreover, the gap between this solution and the best MILP bound is also reported. In the CPU time allowed, the LP model with all 900 small instances is able to find 516 optimum solutions $(57.33 \%)$. Table 6 summarizes the results, which are categorized by factors of solver, threads and time limit. The comparison criteria are: the percentage of optimum solutions found (\%opt), the average gap as a percentage for the cases in which the optimum solution is not found $(G A P \%)$ and the average time required in seconds. Later we will carry out statistical testing to ascertain the significance of the observed differences.

It is clear that GUROBI is able to find more optimal solutions than CPLEX, and its average gap and average CPU time consumption are smaller than CPLEX. Overall, time limit of 3600 seconds and parallel computing (2 threads) results in a larger number of optimal solutions, in comparison with time limit of 900 seconds and serial computing ( 1 thread). CPLEX with parallel computing (2 threads) results in a greater average gap in comparison with serial computing, but this trend is reversed with GUROBI. Among all the eight combinations of test factors, GUROBI with two threads and 3600 seconds time limitation finds more optimum solutions than the others.

\begin{tabular}{cccccc}
\hline \multirow{2}{*}{ Solver } & Time Limit & \multicolumn{2}{c}{$900 s$} & \multicolumn{2}{c}{$3600 s$} \\
\cline { 2 - 6 } & Thread & 1 & 2 & 1 & 2 \\
\hline \multirow{2}{*}{ CPLEX } & $\%$ opt & 59.44 & 61.22 & 63.11 & 61.89 \\
\cline { 2 - 6 } & GAP\% & 29.62 & 30.77 & 32.23 & 36.46 \\
\cline { 2 - 6 } & Av Time (s) & 390.41 & 380.69 & 1426.53 & 1441.80 \\
\hline \multirow{2}{*}{ GUROBI } & $\%$ opt & 66.89 & 68.33 & 70.78 & 73.00 \\
\cline { 2 - 6 } & GAP\% & 2.19 & 2.04 & 1.81 & 1.70 \\
\cline { 2 - 6 } & Av Time (s) & 328.15 & 315.57 & 1152.36 & 1089.00 \\
\hline
\end{tabular}

Table 6: Performance results for solvers, threads and time limit for the small instances.

Automatic Interaction Detection (AID) is an advanced statistical technique for multivariate analysis, which was developed by Morgan and Sonquist (1963). It seeks to find explanatory variables and combinations of these variables which are important for lowering variance in the dependent variables. AID is a stepwise procedure that subdivides experimental data according to one factor through a series of dichotomous splits into a number of mutually exclusive subgroups. The initial AID was improved by Kass (1980) 
by including statistical significance testing in the partition process and by allowing multi-way splits of data resulting in the so-called Chi-squared Automatic Interaction Detection (CHAID). A modification to the basic CHAID algorithm, called an exhaustive CHAID, introduced by Biggs et al. (1991), performs a more thorough merging and testing of factor variables.

An exhaustive CHAID is used to draw a decision tree to analyze the effect and interactions of the factors for the averages observed in Table 6. AID techniques are used in different areas like market research, psychology, education, scheduling, etc. Recently, CHAID was employed by Ruiz et al. (2008) to analyze a complex non distributed scheduling problem MILP model. Also, Ruiz and Andrés-Romano (2011) employed CHAID to analyse a MILP in a problem with unrelated parallel machines with resource-assignable sequencedependent setup times. Naderi and Ruiz (2010) also used CHAID to analyze several models for the distributed permutation flowshop scheduling problem.

The exhaustive CHAID method is used to analyze the MILP results, which were previously presented. The factors, either serial computing or parallel computing (Threads), solver, $n, m, f$ and $t$, are controlled. We introduce all the data of both stopping CPU time criteria so the factor time is controlled as well. The response variable is the type of solution reached by CPLEX and GUROBI with two possible values (0 and 1$)$. We use the PASW statistics version 18 software and set a high confidence level for splitting of $99.9 \%$, as well as a Bonferroni adjustment for the multi-way splits, which compensates the statistical bias in multi-way paired tests.

In Figure 2, the root node contains the total percentage of the cases were instances were solved optimally (type 0 ) and the total number of cases. The most significant factor is the number of jobs or $n$, and the next level is divided into one node for each possible $n$ value. The $p$-value obtained for this split comes very close to 0 and the result of the $\chi^{2}$ statistic is very high, meaning that the split is done with a very high level of confidence; i.e., $n$ is the most influential factor on the response variable with a very statistically significant effect.

Among the resulting five nodes, as the $n$ value increases, the number of cases for which an optimal solution is found decreases. As a matter of fact, for $n=20$ and 24 , only $35.6 \%$ of the instances are optimally solved. After this first multi-way split, nodes are split into the number of factories factor, except for $n=8$. It is logical that when there is a larger amount of factories, jobs have more options for allocations, and the completion time of jobs also shortens. Hence, the earliest possible time to start product assembly also shortens, and 
the possibility of finding a better solution increases. The number of products $t$ is the third next important factor, except for node $n=12 / f=3$, where number of machines is a significant factor. No further statistically significant divisions are found and the stopping criterion for branching is met for nodes $n=12 / f=4$ and $n=24 / f=2$. The number of products factor shows the same trend as the second important factor (number of factories); that is, a higher percentage of optimal solutions is found when there is a larger number of products. If the number of jobs is constant and the number of products increases, fewer jobs will be dedicated to each product on average, so finding a better partial job sequence for each product is easier.

As seen, apart from a few isolated cases, the effect of type of solver, one thread (serial computing) and two threads (parallel computing) and time limit (900s and 3600s) are not statistically significant.

\subsection{Heuristics evaluation}

The twelve proposed methods $\left(\mathrm{H}_{11}, \mathrm{H}_{12}, \mathrm{H}_{21}, \mathrm{H}_{22}, \mathrm{H}_{31}, \mathrm{H}_{32}, \mathrm{VND}_{H_{11}}\right.$, $\mathrm{VND}_{H_{12}}, \mathrm{VND}_{H_{21}}, \mathrm{VND}_{H_{22}}, \mathrm{VND}_{H_{31}}$ and $\left.\mathrm{VND}_{H_{32}}\right)$ are now tested. As the proposed heuristics are not expected to find an optimal solution, the Relative percentage deviation (RPD), is measured for comparisons. We measure $R P D$ as follows: using the optimal solution or the best known solution, which is found through all heuristics and the MILP model $\left(O P T_{\text {best }}\right)$ and $A L G_{S O L}$, which reports the makespan obtained by a given algorithm for a given instance:

$$
R P D=\frac{A L G_{S O L}-O P T_{\text {best }}}{O P T_{\text {best }}} \times 100
$$

Table 7 provides the summarized results of the MILP and the average algorithm deviations from the best known solution for the small instances. They are categorized by $n$ and $f$.

As we can see in Table 7, it is clear that the mathematical model is unable to find an optimum or best solution for all the small instances considered. By increasing the number of jobs $(n)$ and by decreasing number of the factories $(f)$, the problem becomes harder for the MILP to solve. All VND algorithms perform better than the constructive algorithms. $\mathrm{NR}_{2}$ works better than the first one as a rule to assign jobs to factories. In order to know if the differences observed in Table 7 are statistically significant, a multifactor ANOVA of the results of the VND algorithms has to be done. The average RPD value for all the simple constructive heuristics is $6.75 \%$, and this amount lowers to 


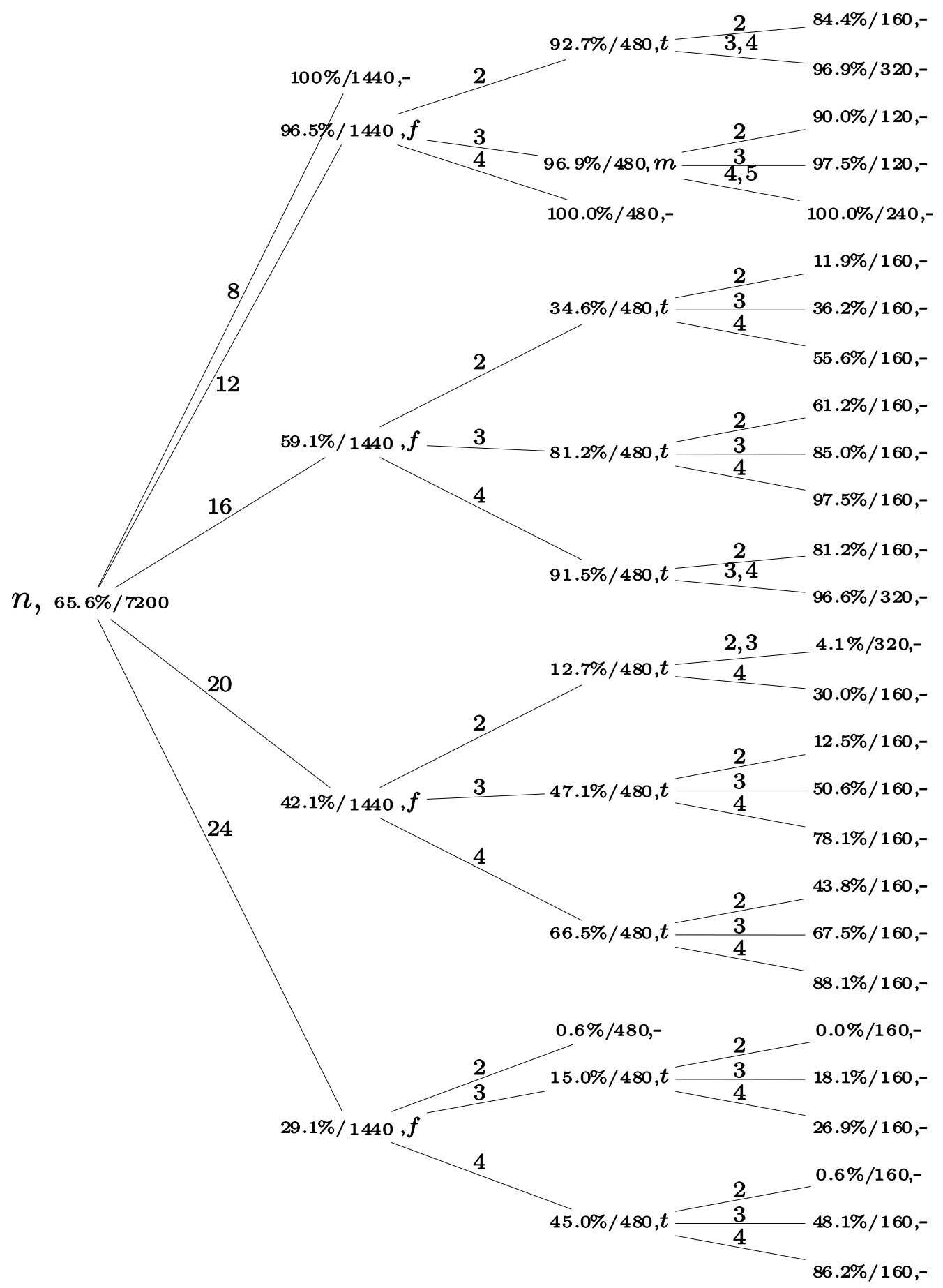

Figure 2: Decision tree for the MILP model evaluation.

19 


\begin{tabular}{|c|c|c|c|c|c|c|c|c|c|c|c|c|c|}
\hline \multirow[b]{2}{*}{$f \times n$} & \multirow[b]{2}{*}{ MILP } & \multicolumn{12}{|c|}{ Algorithms } \\
\hline & & $\vec{F}$ & $\underset{ت}{ت}$ & $\vec{N}$ & $\underset{\mathbb{N}}{\mathbb{N}}$ & $\vec{m}$ & 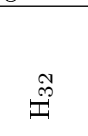 & $\stackrel{I}{Z}_{j}^{\vec{I}}$ & 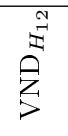 & 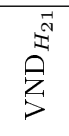 & 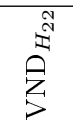 & $\stackrel{\vec{I}}{\vec{n}}_{\vec{j}}^{\vec{n}}$ & 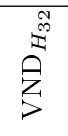 \\
\hline $2 \times 8$ & 0.00 & 14.62 & 13.61 & 6.91 & 5.99 & 13.55 & 12.17 & 1.00 & 0.76 & 1.00 & 0.76 & 1.02 & 0.78 \\
\hline $2 \times 12$ & 0.02 & 13.70 & 12.78 & 5.74 & 5.17 & 11.58 & 11.05 & 0.93 & 0.87 & 0.93 & 0.87 & 0.93 & 0.87 \\
\hline $2 \times 16$ & 0.45 & 12.52 & 11.40 & 5.77 & 5.10 & 10.00 & 9.16 & 0.73 & 0.55 & 0.72 & 0.53 & 1.09 & 0.53 \\
\hline & 1.55 & 10.23 & 9.59 & 4.55 & 3.78 & 8.96 & 8.46 & 0.53 & 0.36 & 0.51 & 0.37 & 0.57 & 0.37 \\
\hline $2 \times 24$ & 3.42 & 8.71 & 8.34 & 5.00 & 4.74 & 7.54 & 7.15 & 0.54 & 0.21 & 0.54 & 0.21 & 0.54 & 0.21 \\
\hline & & & & & & & & 1.09 & 0.70 & 1.15 & 0.76 & 1.15 & 0.76 \\
\hline $3 \times$ & 0.02 & 9.96 & 9.13 & 3.03 & 2.55 & 8.72 & .50 & 0.44 & 0.28 & 0.44 & 0.28 & 0.44 & 0.28 \\
\hline 16 & 0.05 & 10.10 & 9.16 & 3.77 & 3.14 & 9.59 & 8.73 & 0.86 & 0.56 & 0.91 & 0.56 & 0.91 & 0.56 \\
\hline 20 & 0.40 & 9.86 & 8.93 & 2.72 & 2.19 & 8.53 & 7.84 & 0.43 & 0.43 & 0.43 & 0.43 & 0.43 & 0.43 \\
\hline $3 \times 24$ & 1.16 & 7.77 & 6.48 & 3.11 & 2.52 & 7.24 & 6.32 & 0.64 & 0.33 & 0.64 & 0.33 & 0.64 & 0.33 \\
\hline & & & & & & & & 1.08 & 0.63 & 0.99 & 0.63 & 0.99 & 0.63 \\
\hline $4 \times 12$ & 0.00 & 63 & 4.53 & 1.82 & 1.38 & 4.58 & 3.58 & 0.74 & 0.47 & 0.74 & 0.47 & 0.74 & 0.56 \\
\hline & 0.03 & 7.21 & 6.34 & 2.86 & 2.27 & 6.14 & 5.18 & 0.59 & 0.28 & 0.59 & 0.28 & 0.59 & 0.28 \\
\hline & 0.21 & 6.80 & 6.00 & 2.96 & 2.61 & 5.66 & 5.04 & 1.10 & 0.63 & 1.10 & 0.63 & 1.10 & 0.63 \\
\hline $4 \times 24$ & 0.40 & 5.14 & 4.43 & 2.02 & 1.60 & 4.87 & 4.19 & 0.57 & 0.26 & 0.57 & 0.26 & 0.57 & 0.26 \\
\hline inctage & 0.51 & 9.51 & 8.58 & 3.80 & 3.16 & 8.15 & 7.29 & 0.75 & 0.49 & 0.75 & 0.49 & 0.78 & 0.50 \\
\hline
\end{tabular}

Table 7: Relative Percentage Deviation (RPD) of MILP and proposed algorithms over the best known solution for the small instances.

$0.63 \%$ for the VND methods. The RPD factor difference between simple constructive heuristics and VND heuristics is very high. For this reason, we separated the statistical analysis in two ANOVAs: one for the simple heuristics and the other one for the VND methods. As explained before, there are 900 small instances, and each ANOVA considers six simple constructive heuristics or six VND methods with $6 \times 900=5400$ data.

As with all parametric analyses, ANOVA requires some assumptions to be met. These are normality, homocedasticity and independence of residuals. While a slightly strong tailed normal distribution of the residuals is observed, residuals are clearly homoscedastic and independent, and according to the recent results of Basso et al. (2007) and Rasch and Guiard (2004), this is not a major problem. The response factor is again the RPD and the controlled factors are $n, m, f, t$ and algorithms. All the controlled factors in the ANOVA analysis, except $m$ and $t$ in six simple constructive heuristics, and except $f$ factor in six VND methods result in strong statistically significant differences in the RPD response variable, with $p$-values coming very close to zero. The results are not shown here due to reasons of space. In order to identify the best algorithm, the means plot and Tukey's Honest Significant Difference 


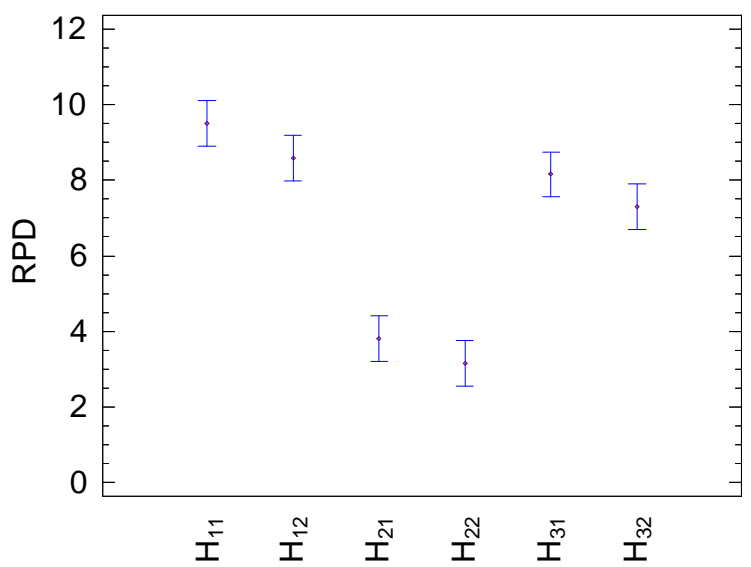

Figure 3: Means plot and 99\% confidence level Tukey's HSD intervals for simple constructive heuristic methods and small instances.

(HSD) intervals (99\% confidence) for the six simple constructive heuristics and VND methods are shown in Figures 3 and 4, respectively.

As it is clear in Figure 3, the second heuristic performs better in comparison with the other simple constructive heuristics and there is no significant differences between the rules used to assign jobs to factories. However, it is obvious in Figure 4 that the rules for allocating jobs to factories are important, and $\mathrm{NR}_{2}$ is statistically different from $\mathrm{NR}_{1}$. It is clear that the VND algorithm almost improves all the initial solutions equally and that the kind of initial solution to start the VND is not important for algorithms with the same job assignment rule. No significant differences between the three VND considered algorithms using $\mathrm{NR}_{2}$ is found.

The CPU times to solve small instances with the considered algorithms are negligible; for example, the $\mathrm{VND}_{H_{32}}$ algorithm with 0.004693 seconds, has the largest average consumed CPU time for the small instances.

\subsection{Heuristics evaluation on large instances}

In this case, for calculating the RPD, the best solution $\left(O P T_{\text {best }}\right)$ is the best solution found among all twelve algorithms because, in large instances, good MILP bounds are not known. A summarized result of the average RPD, considering number of factories, number of products and number of jobs, is shown in Table 8. Algorithms can be categorized into two groups: VND algorithms, $\mathrm{H}_{21}$ and $\mathrm{H}_{22}$, in one group, which perform better, and the rest 


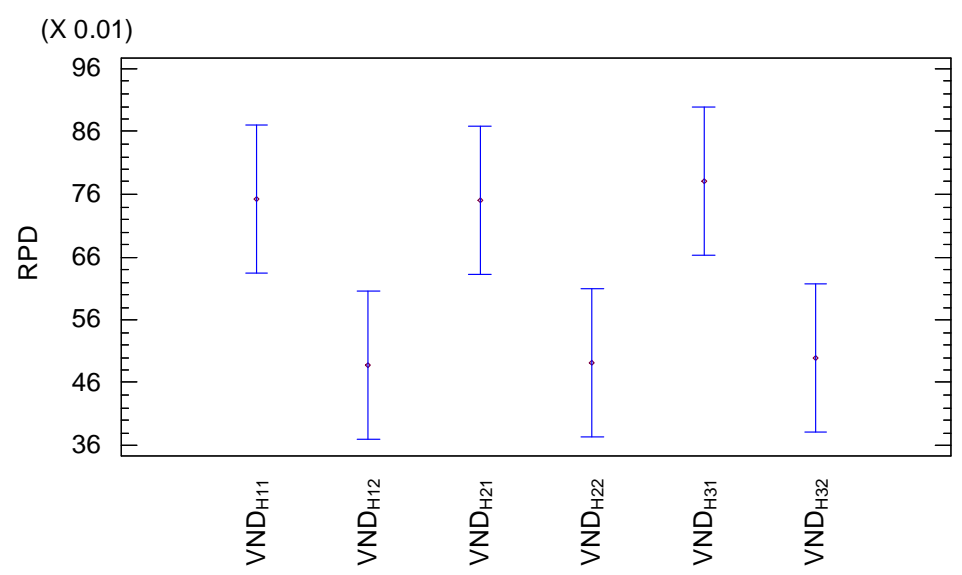

Figure 4: Means plot and 99\% confidence level Tukey's HSD intervals for VND methods and small instances.

in another group. On the other hand, algorithms with $\mathrm{NR}_{2}$ work better than those with $\mathrm{NR}_{1}$.

The second group does not report good results if compared to the first one, so it has been eliminated from the statistical analysis. A multifactorial ANOVA has been carried out with only the first group to know if there are any significant differences between results. Figure 5 shows a means plot $(99 \%$ confidence level Tukey's HSD intervals) for the first group of algorithms. It is clear that the algorithms which use $\mathrm{NR}_{2}$ as a job assignment rule, report better results. Moreover, the type of initial solution for the VND algorithms does not play an important role. Finally, there is no significant difference between the VND algorithms that use the same job allocation rule. 
Algorithms

\begin{tabular}{|c|c|c|c|c|c|c|c|c|c|c|c|c|c|c|c|}
\hline & \multicolumn{13}{|c|}{ Algorithms } \\
\hline & & & $\mathrm{H}_{11}$ & $\mathrm{H}_{12}$ & $\mathrm{H}_{21}$ & $\mathrm{H}_{22}$ & $\mathrm{H}_{31}$ & $\mathrm{H}_{3}$ & & $\mathrm{VND}_{H_{11}}$ & $\mathrm{VND}_{H_{12}}$ & $\mathrm{VND}_{H_{21}}$ & $\mathrm{VND}_{H_{22}}$ & $\mathrm{VND}_{H_{31}}$ & $\mathrm{VND}_{H_{32}}$ \\
\hline \multirow{10}{*}{ 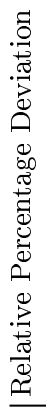 } & \multirow{3}{*}{$\begin{array}{l}\text { Factories } \\
\qquad(f)\end{array}$} & 4 & 5.57 & 5.09 & 0.32 & 0.19 & 2.96 & 2.5 & & 0.06 & 0.03 & 0.05 & 0.01 & 0.05 & 0.01 \\
\hline & & 6 & 3.77 & 3.29 & 0.11 & 0.06 & 1.64 & 1.3 & & 0.03 & 0.01 & 0.02 & 0.00 & 0.02 & 0.00 \\
\hline & & 8 & 3.09 & 2.66 & 0.04 & 0.02 & 1.21 & 0. & & 0.02 & 0.00 & 0.01 & 0.00 & 0.01 & 0.00 \\
\hline & \multirow{3}{*}{$\begin{array}{l}\text { Products } \\
\qquad(t)\end{array}$} & 30 & 3.78 & 3.34 & 0.21 & 0.11 & 2.23 & 1.8 & & 0.03 & 0.01 & 0.04 & 0.01 & 0.04 & 0.01 \\
\hline & & 40 & 4.30 & 3.85 & 0.15 & 0.10 & 1.94 & 1.6 & & 0.04 & 0.02 & 0.02 & 0.01 & 0.02 & 0.01 \\
\hline & & 50 & 4.36 & 3.85 & 0.11 & 0.05 & 1.65 & 1.3 & & 0.04 & 0.01 & 0.02 & 0.00 & 0.02 & 0.00 \\
\hline & \multirow{4}{*}{$\begin{array}{l}\text { Jobs } \\
(n)\end{array}$} & 100 & 6.30 & 5.61 & 0.17 & 0.08 & 2.02 & 1.5 & & 0.05 & 0.02 & 0.03 & 0.01 & 0.03 & 0.01 \\
\hline & & 200 & 3.76 & 3.28 & 0.15 & 0.07 & 1.92 & 1.5 & & 0.03 & 0.01 & 0.02 & 0.00 & 0.02 & 0.00 \\
\hline & & 500 & 2.37 & 2.16 & 0.14 & 0.10 & 1.87 & 1.6 & & 0.03 & 0.01 & 0.03 & 0.01 & 0.03 & 0.01 \\
\hline & & Aver & 4.14 & 3.68 & 0.16 & 0.09 & 1.94 & 1.6 & & 0.04 & 0.01 & 0.03 & 0.01 & 0.03 & 0.01 \\
\hline & \multirow{3}{*}{$\begin{array}{c}\text { Factories } \\
(f)\end{array}$} & 4 & 0.01 & 0.01 & 0.01 & 0.01 & 0.01 & 0.0 & & 4.39 & 6.79 & 2.90 & 7.67 & 2.55 & 42.87 \\
\hline & & 6 & 0.01 & 0.01 & 0.01 & 0.01 & 0.01 & 0.0 & & 3.49 & 7.73 & 2.85 & 8.94 & 1.95 & 6.11 \\
\hline$\dot{0}$ & & 8 & 0.01 & 0.01 & 0.01 & 0.01 & 0.01 & 0.0 & & 3.26 & 9.56 & 1.86 & 10.21 & 1.83 & 20.64 \\
\hline \multirow{7}{*}{ 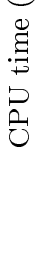 } & \multirow{3}{*}{$\begin{array}{l}\text { Products } \\
\qquad(t)\end{array}$} & 30 & 0.01 & 0.01 & 0.02 & 0.02 & 0.01 & 0.0 & & 3.64 & 8.05 & 3.14 & 11.00 & 2.70 & 45.20 \\
\hline & & 40 & 0.01 & 0.01 & 0.01 & 0.01 & 0.01 & 0.0 & & 3.59 & 7.12 & 2.45 & 8.05 & 1.96 & 5.54 \\
\hline & & 50 & 0.01 & 0.01 & 0.01 & 0.01 & 0.01 & 0.0 & & 3.91 & 8.91 & 2.02 & 7.77 & 1.66 & 18.88 \\
\hline & \multirow{4}{*}{$\begin{array}{c}\text { Jobs } \\
(n)\end{array}$} & 100 & 0.00 & 0.00 & 0.00 & 0.00 & 0.00 & 0.0 & & 1.09 & 2.84 & 0.27 & 0.72 & 0.24 & 0.43 \\
\hline & & 200 & 0.00 & 0.00 & 0.00 & 0.00 & 0.00 & 0.0 & & 2.02 & 3.85 & 0.58 & 2.22 & 0.66 & 1.37 \\
\hline & & 500 & 0.03 & 0.02 & 0.03 & 0.04 & 0.03 & 0.0 & & 8.03 & 17.39 & 6.76 & 23.88 & 5.41 & 67.81 \\
\hline & & Aver & 0.01 & 0.01 & 0.01 & 0.01 & 0.01 & 0.0 & & 3.71 & 8.03 & 2.54 & 8.94 & 2.11 & 23.20 \\
\hline
\end{tabular}

Table 8: Relative Percentage Deviation (RPD) and CPU times of proposed algorithms for the large instances. 


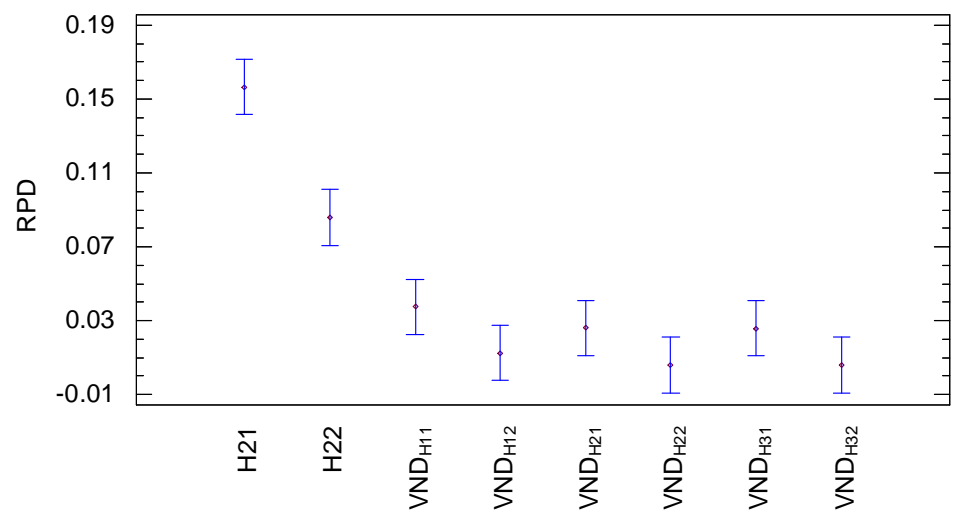

Figure 5: Means plot and 99\% confidence level Tukey's HSD intervals for algorithms and large instances.

518

It is obvious that heuristic 2 performs better than heuristic 3 in both small and large instances.

The interaction between algorithms and $n$ has no significant effect on the response variable. An increase in the number of machines always complicates problems, thus there is no interest in showing these interactions. Interaction between algorithms and the number of factories $f$ is interesting. By increasing the number of factories, the problem becomes easier, as it is shown in Figure 6.

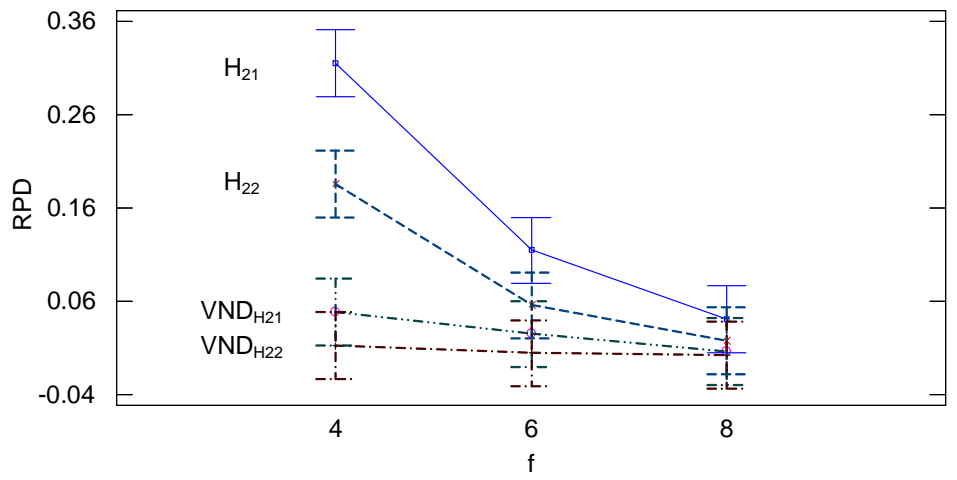

Figure 6: Means plot and 99\% confidence level Tukey's HSD intervals for interaction between algorithms and number of factories $f$ and large instances.

Neither the number of products nor the number of jobs factors have a significant effect, and only an increase in either makes the problem easier to 
solve for simple constructive algorithms. However, neither one has a significant effect on the VND algorithms.

In all the results, the RPD of $\mathrm{VND}_{H_{22}}$ is consistently lower than that of the other algorithms. Thus with more samples, it is expected that it will eventually become statistically better than the others. $\mathrm{VND}_{H_{22}}$ is better than $\mathrm{VND}_{H_{21}}$ because $\mathrm{NR}_{2}$ checks all the factories when assigning a job and finally chooses the best one. It takes longer than $\mathrm{NR}_{1}$, which just places the job at the first available factory. However, when the number of factories increases, the algorithms that use $\mathrm{NR}_{1}$ do not report good results.

The algorithms' CPU time consumption is summarized in Table 8. Simple constructive algorithms use a very short time in order to solve problems, while, as expected, the VND algorithms use more time if compared to simple constructive algorithms. $\mathrm{VND}_{H_{32}}$ consumes an average of 23.20 seconds, the longest CPU time consumption if compared to other algorithms. As Table 8 shows, in the $\mathrm{VND}_{H_{32}}$ algorithm, factors $n=500, t=30$ and $f=4$ are the most CPU time consuming.

The VND methods try to improve the output of simple constructive algorithms and it is logical that take more time than simple constructive algorithms to solve problems. To compensate, VND algorithms report smaller RPD values than simple constructive algorithms. As Table 8 shows, the minimum RPD reported by a simple constructive algorithm is 9 times larger than the largest reported RPD by VND algorithms that use $\mathrm{NR}_{2}$.

If the quality of the solution is more important than $\mathrm{CPU}$ time consumption, then VND algorithms are the best options. Otherwise, a simple constructive algorithm can be a good choice when only CPU time consumption is more important. However, it is worth waiting a maximum time of almost 24 seconds to obtain a good solution. All the experimental results and the best solutions can be found at http://soa.iti.es.

\section{Conclusion and future research}

To the best of our knowledge, this paper is the first attempt to generalize the Distributed Permutation Flowshop Scheduling Problem to the Distributed Assembly Permutation Flowshop Scheduling Problem, where there is more than one production center to process jobs and a single assembly center to make final products from produced jobs. A mathematical model is presented and two solvers are used to solve it. Three constructive algorithms and three VND algorithms are proposed. 
Computational evaluations were performed with two groups of small and large instances, and ANOVAs were used to analyze results. Results show that the VND algorithms report the best results. On the other hand, simple constructive algorithms consume little CPU time and still produce reasonable solutions.

For future works, setup times, transportation stages and distinct factories can be considered for added realism. Other strategies can be used to construct VND neighborhoods. Other metaheuristics may report better solutions if compared to our proposed VND.

\section{Acknowledgements}

Rubén Ruiz is partially supported by the Spanish Ministry of Science and Innovation, under the project "RESULT - Realistic Extended Scheduling Using Light Techniques" with reference DPI2012-36243-C02-01. Carlos Andrés is partially supported by the Spanish Ministry of Science and Innovation, under the project "INSAMBLE - Scheduling at assembly/disassembly synchronized supply chains" with reference DPI2011-27633.

\section{References}

Al-Anzi, F. S., Allahverdi, A., 2006. A hybrid tabu search heuristic for the twostage assembly scheduling problem. The International Journal of Operational Research, 3 (2), 109-119.

Baker, K. R., 1974. Introduction to sequencing and scheduling. Wiley, New York. Basso, D., Chiarandini, M., Salmaso, L., 2007. Synchronized permutation tests inreplicated $i \times j$ designs. Journal of Statistical Planning and Inference, 137 (8), $2564-2578$.

Biggs, D., Ville, B. D., Suen, E., 1991. A method of choosing multiway partitions for classification and decision trees. Journal of Applied Statistics, 18 (1), 49-62.

Chan, F. T. S., Chung, S. H., Chan, P. L. Y., 2005. An adaptive genetic algorithm with dominated genes for distributed scheduling problems. Expert Systems with Applications, 29 (2), 364-371.

Chan, F. T. S., Chung, S. H., Chan, P. L. Y., 2006a. Application of genetic algorithms with dominant genes in a distributed scheduling problem in flexible manufacturing systems. International Journal of Production Research 44 (3), $523-543$.

Chan, F. T. S., Chung, S. H., Chan, P. L. Y., Finke, G., Tiwari, M. K., 2006b. Solving distributed FMS scheduling problems subject to maintenance: genetic algorithms approach. Robotics and Computer-Integrated Manufacturing, 22 (5- 
6), 493-504.

Chen, S. H., Chang, P. C., Cheng, T. C. E., Zhang, Q., 2012. A self-guided genetic algorithm for permutation flowshop scheduling problems. Computers \& Operations Research, 39 (7), 1450-1457.

Ching-Jong, L., Li-Man, L., 2008. Improved MILP models for two-machine flowshop with batch processing machines. Mathematical and Computer Modelling, 48 (78), $1254-1264$.

Dong, X., Huang, H., Chen, P., 2009. An iterated local search algorithm for the permutation flowshop problem with total flowtime criterion. Computers \& Operations Research, 36 (5), 1664-1669.

Framinan, J. M., Leisten, R., 2003. An efficient constructive heuristic for flowtime minimisation in permutation flow shops. Omega, The International Journal of Management Science, 31 (4), 311-317.

Framinan, J. M., Leisten, R., Ruiz-Usano, R., 2002. Efficient heuristics for flowshop sequencing with the objectives of makespan and flowtime minimization. European Journal of Operational Research, 141 (3), 559-569.

Gao, J., Chen, R., 2011a. A hybrid genetic algorithm for the distributed permutation flowshop scheduling problem. International Journal of Computational Intelligence Systems, 4 (4), 497-508.

Gao, J., Chen, R., 2011b. An NEH-based heuristic algorithm for distributed permutation flowshop scheduling problems. Scientific Research and Essays, 6 (14), 3094-3100.

Gao, J., Chen, R., Deng, W., Liu, Y., 2012a. Solving multi-factory flowshop problems with a novel variable neighbourhood descent algorithm. Journal of Computational Information Systems, 8 (5), 2025-2032.

Gao, J., Chen, R., Liu, Y., 2012b. A knowledge-based genetic algorithm for permutation flowshop scheduling problems with multiple factories. International Journal of Advancements in Computing Technology, 4 (7), 121-129.

Hansen, P., Mladenovic, N., 2001. Variable neighborhood search: principles and applications. European Journal of Operational Research, 130 (3), 449-467.

Hariri, A. M. A., Potts, C. N., 1997. A branch and bound algorithm for the twostage assembly scheduling problem. European Journal of Operational Research, $103(2), 547-556$.

Jia, H. Z., Fuh, J. Y. H., Nee, A. Y. C., Zhang, Y. F., 2002. Web-based multifunctional scheduling system for a distributed manufacturing environment. Concurrent Engineering: Research and Applications, 10 (1), 27-39.

Jia, H. Z., Fuh, J. Y. H., Nee, A. Y. C., Zhang, Y. F., 2007. Integration of genetic algorithm and Gantt chart for job shop scheduling in distributed manufacturing systems. Computers \& Industrial Engineering, 53 (2), 313-320.

Jia, H. Z., Nee, A. Y. C., Fuh, J. Y. H., Zhang, Y. F., 2003. A modified genetic algo- 
rithm for distributed scheduling problems. Journal of Intelligent Manufacturing, $14(3-4), 351-362$.

Johnson, S. M., 1954. Optimal two- and three-stage production schedules with setup times included. Naval Research Logistics Quarterly, 1 (1), 61-68.

Kahn, K. B., Castellion, G. A., Griffin, A., 2004. The PDMA handbook of new product development, 2nd Edition. Wiley, New York.

Kass, G. V., 1980. An exploratory technique for investigating large quantities of categorical data. Journal of Applied Statistics, 29 (2), 119-127.

Koulamas, C., Kyparisis, G. J., 2001. The three stage assembly flowshop scheduling problem. Computers \& Operations Research, 28 (7), 689-704.

Laha, D., Sarin, S. C., 2009. A heuristic to minimize total flow time in permutation flow shop. Omega, The International Journal of Management Science, 37 (3), 734-739.

Lee, C. Y., Cheng, T. C. E., Lin, B. M. T., 1993. Minimizing the makespan in the 3 -machine assembly-type flowshop scheduling problem. Management Science, $39(5), 616-625$.

Liu, H., Gao, L., 2010. A discrete electromagnetism-like mechanism algorithm for solving distributed permutation flowshop scheduling problem. International Conference on Manufacturing Automation,, 156-163.

Moon, C., Kim, J., Hur, S., 2002. Integrated process planning and scheduling with minimizing total tardiness in multi-plants supply chain. Computers \& Industrial Engineering, 43 (1-2), 331-349.

Morgan, J. N., Sonquist, J. A., 1963. Problems in the analysis of survey data, and a proposal. Journal of the American Statistical Association, 58 (302), 415-434.

Naderi, B., Ruiz, R., 2010. The distributed permutation flowshop scheduling problem. Computers \& Operations Research, 37 (4), 754-768.

Nawaz, M., Enscore, E. E., Ham, I., 1983. A heuristic algorithm for the m-machine, n-job flowshop sequencing problem. Omega, International Journal of Management Science, 11 (1), 91-95.

Pan, Q. K., Ruiz, R., 2012. Local search methods for the flowshop scheduling problem with flowtime minimization. European Journal of Operational Research, 222 (1), 31-43.

Pan, Q. K., Ruiz, R., 2013. A comprehensive review and evaluation of permutation flowshop heuristics to minimize flowtime. Computers \& Operations Research, 40 (1), 117-128.

Pinedo, M., 2012. Scheduling: Theory, Algorithms and Systems, 4th Edition. Springer, New York.

Potts, C. N., Janov, S. V. S., Strusevich, V. A., Wassenhove, L. N. V., Zwaneveld, C. M., 1995. The two-stage assembly scheduling problem: Complexity and approximation. Operations Research, 43 (2), 346-355. 
Rasch, D., Guiard, V., 2004. The robustness of parametric statistical methods. Psychology Science, 46 (2), 175-208.

Ruiz, R., Andrés-Romano, C., 2011. Scheduling unrelated parallel machines with resource-assignable sequence dependent setup times. International Journal of Advanced Manufacturing Technology, 57 (5-8), 777-794.

Ruiz, R., Şerifoğlu, F. S., Urlings, T., 2008. Modeling realistic hybrid flexible flowshop scheduling problems. Computers \& Operations Research, 35 (4), 1151-1175.

Ruiz, R., Maroto, C., 2005. A comprehensive review and evaluation of permutation flowshop heuristics. European Journal of Operational Research, 165 (2), 479-494.

Ruiz, R., Stützle, T., 2007. A simple and effective iterated greedy algorithm for the permutation flowshop scheduling problem. European Journal of Operational Research, 177 (3), 2033-2049.

Stafford, E. F., Tseng, F. T., Gupta, J. N. D., 2005. Comparative evaluation of MILP flowshop models. Journal of the Operational Research Society, 56 (1), 88-101.

Tozkapan, A., Kirca, O., Chung, C. S., 2003. A branch and bound algorithm to minimize the total weighted flowtime for the two-stage assembly scheduling problem. Computers \& Operations Research, 30 (2), 309-320.

Tseng, F. T., Stafford, E. F., 2008. New MILP models for the permutation flowshop problem. Journal of the Operational Research Society, 59 (10), 1373-1386.

Vallada, E., Ruiz, R., 2010. Genetic algorithms with path relinking for the minimum tardiness permutation flowshop problem. Omega, The International Journal of Management Science, 38 (1-2), 57-67.

Vallada, E., Ruiz, R., Minella, G., 2008. Minimising total tardiness in the mmachine flowshop problem: A review and evaluation of heuristics and metaheuristics. Computers \& Operations Research, 35 (4), 1350-1373.

Vollmann, T. E., Berry, W. L., Whybark, D. C., Jacobs, F. R., 2005. Manufacturing Planning and Control for Supply Chain Management, 5th Edition. McGraw-Hill, Boston.

Wang, B., 1997. Integrated product, process and enterprise design, 1st Edition. Chapman \& Hall, London.

Xu, X., Xu, Z., Gu, X., 2011. An asynchronous genetic local search algorithm for the permutation flowshop scheduling problem with total flowtime minimization. Expert Systems with Applications, 38 (7), 7970-7979.

Zhang, Y., Li, X., 2011. Estimation of distribution algorithm for permutation flow shops with total flowtime minimization. Computers \& Industrial Engineering, 60 (4), 706-718.

Zobolas, G. I., Tarantilis, C. D., Ioannou, G., 2009. Minimizing makespan in permutation flowshop scheduling problems using a hybrid metaheuristic algorithm. Computers \& Operations Research, 36 (4), 1249-1267. 